\title{
$\eta$-RICCI SOLITONS AND GRADIENT RICCI SOLITONS ON $\delta$ - LORENTZIAN TRANS-SASAKIAN MANIFOLDS
}

\author{
Mohd Danish Siddiqi ${ }^{1}$ and Mehmet Akif $\mathbf{A k y o l}^{2}$ \\ ${ }^{1}$ Faculty of Science, Department of Mathematics, Jazan University, \\ Jazan, Kingdom of Saudi Arabia \\ ${ }^{2}$ Faculty of Science and Arts, Department of Mathematics, 12000 Bingöl, Turkey
}

\begin{abstract}
The objective of the present research article is to study the $\delta$-Lorentzian trans-Sasakian manifolds concidering the $\eta$-Ricci solitons and gradient Ricci soliton. We have shown that a symmetric second order covariant tensor in a $\delta$-Lorentzian transSasakian manifold is a constant multiple of metric tensor. Also, we have provided an example of $\eta$-Ricci soliton on 3-diemsional $\delta$-Lorentzian trans-Sasakian manifold in the region where $\delta$-Lorentzian trans-Sasakian manifold is expanding. Furthermore, we have discussed the results based on gradient Ricci solitons on 3-dimensional $\delta$ - Lorentzian trans-Sasakian manifold.
\end{abstract}

Keywords: $\eta$-Ricci Soliton, Gradient Ricci Soliton, $\delta$-Lotentzian trans-Sasakian manifolds, Einstein manifolds

\section{Introduction}

In geometrical analysis, a differentiable manifolds endowed Lorentzian metric having signature $(-,+,+, \cdots,+)$ is a absolutely fascinating topic in Lorentzian geometry. Matsumoto [19] popularized the study of Lorentzian para-contact manifolds with Lorentzian metric. Ikawa and Erdogan [16] discussed Lorentzian Sasakian manifold. In [38], Yildiz et al. studied Lorentzian $\alpha$-Sasakian manifold and Lorentzian $\beta$ Kenmotsu manifold studied by Funda et al. in [37]. After that, Pujar and Khairnar

Received October 10, 2020. accepted December 10, 2020.

Communicated by Uday Chand De

Corresponding Author: Mohd Danish Siddiqi, Faculty of Science, Department of Mathematics, Jazan University, Jazan, Kingdom of Saudi Arabia | E-mail: msiddiqi@jazanu.edu.sa 2010 Mathematics Subject Classification. Primary 53C15, 53C25; Secondary 53C55, 53D25

(C) 2021 by University of Niš, Serbia | Creative Commons License: CC BY-NC-ND 
[22] have initiated the notion of Lorentzian trans-Sasakian manifolds and studied some basic results with some of its properties. Before that, Pujar had initiated the study of $\delta$-Lorentzian $\alpha$-Sasakian manifolds and $\delta$-Lorentzian $\beta$-Kenmotsu manifolds ([22], [23]). In [11], De also studied properties of curvatures in Lorentzian trans-Sasakian manifolds which is closely related to this subject.

The interplay between manifolds and indefinite metrics is of interest from the overview of physics and relativity. In 1969, Takahashi [32] introduced the notion of almost contact metric manifolds equipped with pseudo-Riemannian metric. These indefinite almost contact metric manifolds and indefinite Sasakian manifolds are known as $(\epsilon)$-almost contact metric manifolds [36]. The concept of $(\epsilon)$-Sasakian manifolds was initiated by Bejancu and Duggal [3]. De and Sarkar [9] studied the notion of $(\epsilon)$-Kenmotsu manifolds. Shukla and Singh [25] extended the study to $(\epsilon)$ trans-Sasakian manifolds with indefinite metric. The semi-Riemannian manifolds has the index 1 and the structure vector field $\xi$ is always a timelike. This motivated the Tripathi et al. [33] to introduce $(\epsilon)$-almost para contact structure where the vector field $\xi$ is spacelike or timelike according to $(\epsilon)=1$ or $(\epsilon)=-1$.

If $M$ has a Lorentzian metric $g$, that is, a symmetric non degenerate $(0,2)$ tensor field of index 1 , then $M$ is called a Lorentzian manifold. Since the Lorentzian metric is of index 1, Lorentzian manifold $M$ has not only spacelike vector fields but also timelike and lightlike vector fields. This difference with the Riemannian case give interesting properties on the Lorentzian manifold. A differentiable manifold $M$ has a Lorentzian metric if and only if $M$ has a 1-dimensional distribution. Since odd dimensional manifold is able to have a Lorentzian metric. Inspired from the previous results, Bhati [1] developed the notion of $\delta$-Lorentzian trans-Sasakian manifolds.

On the other hand, in 1982, Hamilton [14] introduced that the Ricci solitons move under the Ricci flow simply by diffeomorphisms of the initial metric, which means they are stationary points of the Ricci flow is given by

$$
\frac{\partial g}{\partial t}=-2 S(g) .
$$

Definition 1.1. A Ricci soliton $(g, V, \lambda)$ on a Riemannian manifold is defined by

$$
L_{V} g+2 S+2 \lambda=0,
$$

where $S$ is the Ricci tensor, $L_{V}$ is the Lie derivative along the vector field $V$ on $M$ and $\lambda$ is a real scalar. Ricci soliton is said to be shrinking, steady or expanding according as $\lambda<0, \lambda=0$ and $\lambda>0$, respectively.

If the vector field $\mathrm{V}$ is the gradient of a potential function $\psi$, then $g$ is called a gradient Ricci soliton and equation 1.2 assumes the form $\nabla \nabla \psi=S+\lambda g$.

The roots of contact geometry lie in differential equations as in 1872 Sophus Lie introduced the notion of contact transformation as a geometric tool to study systems of differential equations. This subject has manifold connections with the other fields of pure mathematics, and substantial applications in applied areas such as mechanics, optics, phase space of dynamical system, thermodynamics and control theory. 
In 1925, Levy [17] obtained the necessary and sufficient conditions for the existence of such tensors. Later on, R. Sharma [24] initiated the study of Ricci solitons in contact Riemannian geometry. Bagewadi et al. [15] extensively studied Ricci soliton in almost $(\epsilon, \delta)$-trans-Sasakian manifolds. In 2009, Cho and Kimura [8] introduced the notion of $\eta$-Ricci solitons and gave a classification of real hypersurfaces in nonflat complex space forms admitting $\eta$-Ricci solitons. In addition, $\eta$-Ricci solitons with various structures have been studied by various geometers such as Calin and Crasmareanu [7] and Blaga ([4], [5]). Recently, Venu at al. [35] study the $\eta$-Ricci soliton in trans-Sasakian manifold. The first author of the paper also studied some properties of $\eta$-Ricci solitons on $(\epsilon, \delta)$-trans-Sasakian manifold and normal almost contact manifolds which is merely connected to this topic (for more details see [27], [28], [29], [30], [31]). Therefore, it is natural and interesting to study $\eta$-Ricci soliton on $\delta$-Lorentzian trans-Sasakian manifolds. In this paper, we derive the condition for a 3 dimensional $\delta$-Lorentzian trans-Sasakian manifold whose metric as an $\eta$-Ricci soliton and derive expression for the scalar curvature.

\section{Preliminaries}

Let $M$ be an $\delta$-almost contact metric manifold equipped with $\delta$-almost contact metric structure $(\phi, \xi, \eta, g, \delta)$ consisting of a $(1,1)$ tensor field $\phi$, a vector field $\xi$, a 1-form $\eta$ and an indefinite metric $g$ such that

$$
\phi^{2}=X+\eta(X) \xi, \quad \eta(\xi)=-1, \quad \eta \circ \phi=0, \quad \phi \xi=0,
$$

for all $X, Y \in M$, where $\delta$ is such that $\delta^{2}=1$ so that $\delta= \pm 1$. The above structure $(\phi, \xi, \eta, g, \delta)$ on $M$ is called the $\delta$-Lorentzian structure on $M$. If $\delta=1$ and this is usual Lorentzian structure [34] on $M$, the vector field $\xi$ is the timelike that is $M$ contains a timelike vector field. In [34], Tanno classified the connected almost contact metric manifold. For such a manifold the sectional curvature of the plane section containing $\xi$ is constant, say $c$. He showed that they can be divided into three classes. (1) homogeneous normal contact Riemannian manifolds with $c>0$. Other two classes can be seen in Tanno [34].

Gray and Harvella [13] introduced the classification of almost Hermitian manifolds, there appears a class $W_{4}$ of Hermitian manifolds which are closely related to the conformal Kahler manifolds. The class $C_{6} \oplus C_{5}[13]$ coincides with the class of trans-Sasakian structure of type $(\alpha, \beta)$. In fact, the local nature of the two sub classes, namely $C_{6}$ and $C_{5}$ of trans-Sasakian structures are characterized completely.

An almost contact metric structure on $M$ is called a trans-Sasakian [21] if $(M \times \mathbb{R}, J, G)$ belongs to the class $W_{4}$, where $J$ is almost complex structure on $M \times \mathbb{R}$ defined by

$$
J\left(X, \psi \frac{d}{d t}\right)=\left(\phi(X)-\psi \xi, \eta(X) \frac{d}{d t}\right)
$$


for all vector fields $X$ on $M$ and smooth functions $\psi$ on $M \times \mathbb{R}$ and $G$ is the product metric on $M \times \mathbb{R}$. This may be expressed by the condition

$$
\left(\nabla_{X} \phi\right) Y=\alpha(g(X, Y) \xi-\eta(Y) X)+\beta(g(\phi X, Y) \xi-\eta(Y) \phi X)
$$

for any vector fields $X$ and $Y$ on $M, \nabla$ denotes the Levi-Civita connection with respect to $g, \alpha$ and $\beta$ are smooth functions on $M$. The existence of condition (2.3) is ensure by the above discussion.

With the above literature now we define the $\delta$-Lorentzian trans-Sasakian manifolds as follows.

Definition 2.1. A $\delta$-Lorentzian manifold with structure $(\phi, \xi, \eta, g, \delta)$ is said to be $\delta$-Lorentzian trans-Sasakian manifold of type $(\alpha, \beta)$ if it satisfies the condition

$$
\left(\nabla_{X} \phi\right) Y=\alpha(g(X, Y) \xi-\delta \eta(Y) X)+\beta(g(\phi X, Y) \xi-\delta \eta(Y) \phi X)
$$

for any vector fields $X$ and $Y$ on $M$.

If $\delta=1$, then the $\delta$-Lorentzian trans-Sasakian manifold is the usual Lorentzian trans-Sasakian manifold of type $(\alpha, \beta)[11]$. $\delta$-Lorentzian trans-Sasakian manifold of type $(0,0),(0, \beta)(\alpha, 0)$ are the Lorentzian cosymplectic, Lorentzian $\beta$-Kenmotsu and Lorentzian $\alpha$-Sasakian manifolds respectively. In particular if $\alpha=1, \beta=0$ and $\alpha=0, \beta=1$, the $\delta$-Lorentzian trans-Sasakian manifolds reduces to $\delta$-Lorentzian Sasakian and $\delta$-Lorentzian Kenmotsu manifolds respectively.

From (2.4), we have

$$
\nabla_{X} \xi=\delta\{-\alpha \phi(X)-\beta(X+\eta(X) \xi\},
$$

and

$$
\left(\nabla_{X} \eta\right) Y=\alpha g(\phi X, Y)+\beta[g(X, Y)+\delta \eta(X) \eta(Y)] .
$$

In a $\delta$-Lorentzian trans-Sasakian manifold $M$, we have the following relations:

$$
\begin{gathered}
\begin{array}{c}
(2.7) \quad R(X, Y) \xi=\left(\alpha^{2}+\beta^{2}\right)[\eta(Y) X-\eta(X) Y]+2 \alpha \beta[\eta(Y) \phi X-\eta(X) \phi Y] \\
+\delta\left[(Y \alpha) \phi X-(X \alpha) \phi Y+(Y \beta) \phi^{2} X-(X \beta) \phi^{2} Y\right]
\end{array} \\
(2.8) \quad S(X, \xi)=\left[\left((n-1)\left(\alpha^{2}+\beta^{2}\right)-(\xi \beta)\right] \eta(X)+\delta((\phi X) \alpha)+(n-2) \delta(X \beta),\right. \\
\left.(2.9) \quad Q \xi=\delta(n-1)\left(\alpha^{2}+\beta^{2}\right)-(\xi \beta)\right) \xi+\delta \phi(\operatorname{grad} \alpha)-\delta(n-2)(\operatorname{grad} \beta),
\end{gathered}
$$

where $R$ is curvature tensor, while $Q$ is the Ricci operator given by $S(X, Y)=$ $g(Q X, Y)$.

Further in an $\delta$-Lorentzian trans-Sasakian manifold, we have

$$
\delta \phi(\operatorname{grad} \alpha)=\delta(n-2)(\operatorname{grad} \beta)
$$

and

$$
2 \alpha \beta-\delta(\xi \alpha)=0 .
$$


By using (2.7) and (2.10), for constants $\alpha$ and $\beta$, we have

$$
\begin{gathered}
R(\xi, X) Y=\left(\alpha^{2}+\beta^{2}\right)[\delta g(X, Y) \xi-\eta(Y) X], \\
R(X, Y) \xi=\left(\alpha^{2}+\beta^{2}\right)[\eta(Y) X-\eta(X) Y], \\
\eta(R(X, Y) Z)=\delta\left(\alpha^{2}+\beta^{2}\right)[g(Y, Z) \eta(X)-g(X, Z) \eta(Y)], \\
S(X, \xi)=\left[\left((n-1)\left(\alpha^{2}+\beta^{2}\right)-\delta(\xi \beta)\right] \eta(X),\right. \\
Q \xi=\left[(n-1)\left(\alpha^{2}+\beta^{2}\right)-(\xi \beta)\right] \xi .
\end{gathered}
$$

An important consequence of (2.5) is that $\xi$ is a geodesic vector field

$$
\nabla_{\xi} \xi=0 .
$$

For arbitrary $X$ vector field, we have that

$$
d \eta(\xi, X)=0 .
$$

The $\xi$-sectional curvature $K_{\xi}$ of $M$ is the sectional curvature of the plane spanned by $\xi$ and a unit vector field $X$. From (2.13), we have

$$
K_{\xi}=g(R(\xi, X), \xi, X)=\left(\alpha^{2}+\beta^{2}\right)-\delta(\xi \beta) .
$$

It follows from (2.19) that $\xi$-sectional curvature does not depend on $X$.

\section{3. $\quad \eta$-Ricci solitons on $(M, \phi, \xi, \eta, g, \delta)$}

Fix $h$ a symmetric tensor field of (0,2)-type which we suppose to be parallel with respect to the Levi-Civita connection $\nabla$ that is $\nabla h=0$. Applying the Ricci commutation identity [12],

$$
\nabla^{2} h(X, Y ; Z, W)-\nabla^{2} h(X, Y ; W, Z)=0,
$$

we obtain the relation

$$
h(R(X, Y) Z, W)+h(Z, R(X, Y) W)=0 .
$$

Replacing $Z=W=\xi$ in (3.2) and using (2.7) and also use the symmetry of $h$, we have

$$
\begin{array}{r}
\left(3.32\left(\alpha^{2}+\beta^{2}\right)[\eta(Y) h(X, \xi)-\eta(X) h(Y, \xi)]+2 \delta[(Y \alpha) h(\phi X, \xi)-(X \alpha) h(\phi Y, \xi)]\right. \\
+2 \delta\left[(Y \beta) h\left(\phi^{2} X, \xi\right)-(X \beta) h\left(\phi^{2} Y, \xi\right)\right]+4 \alpha \beta[\eta(Y) h(\phi X, \xi)-\eta(X) h(\phi Y, \xi)] .
\end{array}
$$

Adopting $X=\xi$ in (3.3) and by virtue of (2.1), we turn up $(3.4)-2\left[(\delta \xi \alpha-2 \alpha \beta] h(\phi Y, \xi)+2\left[\left(\alpha^{2}+\beta^{2}\right)-\delta(\xi \beta)\right][\eta(Y) h(\xi, \xi)-h(Y, \xi)]=0\right.$. 
By adopting (2.11) in (3.4), we have

$$
\left[\left(\alpha^{2}+\beta^{2}\right)-\delta(\xi \beta)\right][\eta(Y) h(\xi, \xi)-h(Y, \xi)]=0 .
$$

Suppose $\left(\alpha^{2}+\beta^{2}\right)-\delta(\xi \beta) \neq 0$, it results

$$
h(Y, \xi)=\eta(Y) h(\xi, \xi) .
$$

Now, we call a regular $\delta$-Lorentzian trans-Sasakian manifold with $\left(\alpha^{2}+\beta^{2}\right)-$ $\delta(\xi \beta) \neq 0$, where regularity, means the non-vanishing of the Ricci curvature with respect to the generator of $\delta$-Lorentzian trans-Sasakian manifolds.

Differentiating (3.6) covariantly with respect to $X$, we have

$$
\begin{aligned}
(3.7)\left(\nabla_{X} h\right)(Y, \xi)+h\left(\nabla_{X} Y, \xi\right)+h\left(Y, \nabla_{X} \xi\right)= & {\left[\delta g\left(\nabla_{X} Y, \xi\right)+\delta g\left(Y, \nabla_{X} \xi\right)\right] h(\xi, \xi) } \\
& +\eta(Y)\left[\left(\nabla_{X} h\right)(Y, \xi)+2 h\left(\left(\nabla_{X} \xi, \xi\right)\right] .\right.
\end{aligned}
$$

By adopting the parallel condition $\nabla h=0, \eta\left(\nabla_{X} \xi\right)=0$ and by the virtue of (3.6) in $(3.7)$, we get

$$
h\left(Y, \nabla_{X} \xi\right)=\delta g\left(Y, \nabla_{X} \xi\right) h(\xi, \xi) .
$$

Now adopting (2.5) in the above equation, we turn up

$$
-\alpha h(Y, \phi X)+\beta \delta h(Y, X)=-\alpha g(Y, \phi X) h(\xi, \xi)+\beta \delta g(Y, X) h(\xi, \xi) .
$$

Replacing $X=\phi X$ in (3.8) and after simplification, we turn up

$$
h(X, Y)=\delta g(X, Y) h(\xi, \xi),
$$

which together with the standard fact that the parallelism of $h$ implies that $h(\xi, \xi)$ is a constant, via (3.6). Now by considering the above equations, we can gives the conclusion:

Theorem 3.1. Let $(M, \phi, \xi, \eta, g, \delta)$ be an $\delta$-Lorentzian trans-Sasakian manifold with non-vanishing $\xi$-sectional curvature and endowed with a tensor field $h \in \Gamma\left(T_{2}^{0}(M)\right)$ which is symmetric and $\phi$-skew-symmetric. If $h$ is parallel with respect to $\nabla$ then it is a constant multiple of the metric tensor $g$.

Definition 3.1. Let $(M, \phi, \xi, \eta, g, \delta)$ be an $\delta$-almost contact metric manifold. Consider the equation

$$
L_{\xi} g+2 S+2 \lambda g+2 \mu \eta \otimes \eta=0
$$

where $L_{\xi}$ is the Lie derivative operator along the vector field $\xi, S$ is the Ricci curvature tensor field of the metric $g$ and $\lambda$ and $\mu$ are real constants. Writing $L_{\xi} \mathrm{g}$ in terms of the Levi-Civita connection $\nabla$, we obtain:

$$
2 S(X, Y)=-g\left(\nabla_{X} \xi, Y\right)-g\left(X, \nabla_{X} \xi\right)-2 \lambda g(X, Y)-2 \mu \eta(X) \eta(Y),
$$

for any $X, Y \in \chi(M)$.

The data $(g, \xi, \lambda, \mu)$ which satisfies the equation (3.10) is said to be $\eta$-Ricci soliton on $M$ [5]; in particular if $\mu=0$ then $(g, \xi, \lambda)$ is Ricci soliton [5] and its called shrinking, steady or expanding according as $\lambda<0, \lambda=0$ or $\lambda>0$ respectively [5]. 
Now, from (2.5), the equation (3.10) becomes:

$$
S(X, Y)=-(\lambda+\beta \delta) g(X, Y)+(\beta \delta-\mu) \eta(X) \eta(Y) .
$$

The above equations yields

$$
\begin{gathered}
S(X, \xi)=-(\lambda+\mu) \eta(X) \\
Q X=-(\lambda+\beta \delta) X+(\beta \delta-\mu) \xi \\
Q \xi=-(\lambda+\mu) \xi \\
r=-\lambda n-(n-1) \beta \delta-\mu,
\end{gathered}
$$

where $r$ is the scalar curvature. Of the two natural situations regarding the vector field $V$ such that $V \in \operatorname{span}\{\xi\}$ and $V \perp \xi$, we investigate only the case for $V=\xi$.

Our interest is in the expression for $L_{\xi} g+2 S+2 \mu \eta \otimes \eta$. A direct computation gives

$$
L_{\xi} g(X, Y)=2 \beta \delta[g(X, Y)+\eta(X) \eta(Y)] .
$$

In 3 -dimensional $\delta$-Lorentzian trans-Sasakian manifold the Riemannian curvature tensor is given by

$$
\begin{gathered}
R(X, Y) Z=g(Y, Z) Q X-g(X, Z) Q Y+S(Y, Z) X-S(X, Z) Y \\
-\frac{r}{2}[g(Y, Z) X-g(X, Z) Y] .
\end{gathered}
$$

Putting $Z=\xi$ in (3.18) and using (2.7) and (2.8) for 3-dimensional $\delta$-Lorentzian trans-Sasakian manifold, we get

$$
\begin{aligned}
&\left(\alpha^{2}+\beta^{2}\right)[\eta(Y) X-\eta(X) Y]+2 \alpha \beta[\eta(Y) \phi X-\eta(X) \phi Y] \\
&+\delta[(Y \alpha) \phi X-(X \alpha) \phi Y]+\delta\left[(Y \beta) \phi^{2} X-(X \beta) \phi^{2} Y\right] \\
&= {\left[\left(\alpha^{2}+\beta^{2}\right)-(\xi \beta)\right][\eta(Y) X-\eta(X) Y] } \\
&+\delta \eta(Y) Q X-\delta \eta(X) Q Y-\delta[((\phi Y) \alpha) X+(Y \beta) X] \\
&+\delta[((\phi X) \alpha) Y+(X \beta) Y] .
\end{aligned}
$$

Again, putting $Y=\xi$ in the (3.19) and using (2.1) and (2.11), we turn up

$$
Q X=\left[\frac{r}{2}+(\xi \beta)-\left(\alpha^{2}+\beta^{2}\right)\right] X+\left[\frac{r}{2}+(\xi \beta)-3\left(\alpha^{2}+\beta^{2}\right)\right] \eta(X) \xi .
$$

From (3.20), we have

$$
S(X, Y)=\left[\frac{r}{2}+(\xi \beta)-\left(\alpha^{2}+\beta^{2}\right)\right] g(X, Y)
$$




$$
+\left[\frac{r}{2}+(\xi \beta)-3\left(\alpha^{2}+\beta^{2}\right)\right] \delta \eta(X) \eta(Y) .
$$

Equation (3.21) shows that a 3 -dimensional $\delta$-Lorentzian trans-Sasakian manifold is $\eta$-Einstein.

Next, we consider the equation

$$
h(X, Y)=\left(L_{\xi} g\right)(X, Y)+2 S(X, Y)+2 \mu \eta(X) \eta(Y) .
$$

By using (3.17) and (3.21) in (3.22), we have

$$
\begin{aligned}
& h(X, Y)=\left[r-4\left(\alpha^{2}+\beta^{2}\right)+2 \beta \delta\right] g(X, Y) \\
& \quad+\left[8\left(\alpha^{2}+\beta^{2}\right)-2 \beta \delta-r\right] \delta \eta(X) \eta(Y)+2 \mu \eta(X) \eta(Y) .
\end{aligned}
$$

Setting $X=Y=\xi$ in $(2.3)$, we turn up

$$
h(\xi, \xi)=2\left[2 \delta\left(\alpha^{2}+\beta^{2}\right)-2 \mu\right] .
$$

Now, (3.9) becomes

$$
h(X, Y)=2\left[2 \delta\left(\alpha^{2}+\beta^{2}\right)-2 \mu\right] \delta g(X, Y) .
$$

From (3.22) and (3.25), it follows that $g$ is an $\eta$-Ricci soliton. Therefore, we can state as:

Theorem 3.2. Let $(M, \phi, \xi, \eta, g, \delta)$ be a 3-dimensional $\delta$-Lorentzian trans-Sasakian manifold, then $(g, \xi, \mu)$ yields an $\eta$-Ricci soliton on $M$.

Let $V$ be pointwise collinear with $\xi$. i.e., $V=b \xi$, where $b$ is a function on the 3 -dimensional $\delta$-Lorentzian trans-Sasakian manifold. Then

$$
g\left(\nabla_{X} b \xi, Y\right)+g\left(\nabla_{Y} b \xi, X\right)+2 S(X, Y)+2 \lambda g(X, Y)+2 \mu \eta(X) \eta(Y)=0
$$

or

$$
\begin{gathered}
b g\left(\left(\nabla_{X} \xi, Y\right)+(X b) \eta(Y)+b g\left(\nabla_{Y} \xi, X\right)+(Y b) \eta(X)\right. \\
+2 S(X, Y)+2 \lambda g(X, Y)+2 \mu \eta(X) \eta(Y)=0 .
\end{gathered}
$$

By using (2.5), we obtain

$$
\begin{gathered}
b g(-\delta \alpha \phi X-\beta \delta(X+\eta(X) \xi, Y)+(X b) \eta(Y)+b g(-\delta \alpha \phi Y-\beta \delta(Y+\eta(Y) \xi, X) \\
+(Y b) \eta(X)+2 S(X, Y)+2 \lambda g(X, Y)+2 \mu \eta(X) \eta(Y)=0
\end{gathered}
$$

which yields

$$
-2 b \beta \delta g(X, Y)-2 b \beta \delta \eta(X) \eta(Y)+(X b) \eta(Y)
$$




$$
+(Y b) \eta(X)+2 S(X, Y)+2 \lambda g(X, Y)+2 \mu \eta(X) \eta(Y)=0 .
$$

Replacing $Y$ by $\xi$ in (3.26), we get

$$
(X b)+(\xi b) \eta(X)+2\left[2\left(\alpha^{2}+\beta^{2}\right)-(\xi \beta)+\lambda+\mu-2 b \beta \delta\right] \eta(X) .
$$

Again putting $X=\xi$ in (3.27), we obtain

$$
\xi b=-2\left(\alpha^{2}+\beta^{2}\right)+(\xi \beta)-\lambda-\mu+2 b \beta \delta .
$$

Plugging this in (3.27), we get

$$
(X b)+2\left[2\left(\alpha^{2}+\beta^{2}\right)-(\xi \beta)+\lambda+\mu-2 b \beta \delta\right] \eta(X)=0
$$

or

$$
d b=-\left\{\lambda+\mu-(\xi \beta)+2\left(\alpha^{2}+\beta^{2}\right)-2 b \beta \delta\right\} \eta .
$$

Applying $d$ on (3.28), we get $\left\{\lambda+\mu-(\xi \beta)+2\left(\alpha^{2}+\beta^{2}\right)-2 b \beta \delta\right\} d \eta$. Since $d \eta \neq 0$ we have

$$
\lambda+\mu-(\xi \beta)+2\left(\alpha^{2}+\beta^{2}\right)-2 b \beta \delta=0 .
$$

Equation (3.29) in (3.28) yields $b$ as a constant. Therefore, from (3.26), it follows that

$$
S(X, Y)=-(\lambda+2 b \beta \delta) g(X, Y)+(2 b \beta \delta-\mu) \eta(X) \eta(Y),
$$

which implies that $M$ is of constant scalar curvature for constant $2 \beta \delta$. This leads to the following:

Theorem 3.3. If in a 3-dimensional $\delta$-Lorentzian trans-Sasakian manifold the metric $g$ is an $\eta$-Ricci soliton and $V$ is positive collinear with $\xi$, then $V$ is a constant multiple of $\xi$ and $g$ is of constant scalar curvature provided $\beta \delta$ is a constant.

Ranking $X=Y=\xi$ in (3.9) and (3.21) and comparing, we get

$$
\lambda=-2\left(\alpha^{2}+\beta^{2}\right)-(\xi \beta)+\mu-2 b \beta \delta=-2 K_{\xi}-\mu .
$$

From (3.16) and (3.30), we obtain

$$
r=6\left(\alpha^{2}+\beta^{2}\right)-3(\xi \beta)-2 \beta \delta+2 \mu .
$$

Since $\lambda$ is a constant, it follows from (3.30) that $K_{\xi}$ is a constant.

Theorem 3.4. Let $(g, \xi, \mu)$ be an $\eta$-Ricci soliton in $(M, \phi, \xi, \eta, g, \delta)$ a 3-dimensional $\delta$-Lorentzian trans Sasakian manifold. Then the scalar $\lambda+\mu=-2 K_{\xi}, r=6 K_{\xi}+$ $2 \mu-3(\xi \beta)-2 b \beta \delta$.

Remark 3.1. For $\mu=0$, (3.30) reduces to $\lambda=-2 K_{\xi}$, so Ricci soliton in 3-dimensional $\delta$-Lorentzian trans-Sasaakian manifold is shrinking. 
Example 3.1. Consider the three dimensional manifold $M=\left\{(x, y, z) \in \mathbb{R}^{3}|z \neq 0|\right\}$, where $(x, y, z)$ are the Cartesian coordinates in $\mathbb{R}^{3}$ and let the vector fields are

$$
e_{1}=\frac{e^{x}}{z^{2}} \frac{\partial}{\partial x}, \quad e_{2}=\frac{e^{y}}{z^{2}} \frac{\partial}{\partial y}, \quad e_{3}=\frac{-(\delta)}{2} \frac{\partial}{\partial z},
$$

where $e_{1}, e_{2}, e_{3}$ are linearly independent at each point of $M$. Let $g$ be the Riemannain metric defined by

$g\left(e_{1}, e_{1}\right)=g\left(e_{2}, e_{2}\right)=g\left(e_{3}, e_{3}\right)=-\delta, g\left(e_{1}, e_{3}\right)=g\left(e_{2}, e_{3}\right)=g\left(e_{1}, e_{2}\right)=0$, where $\delta$ is such that $\delta^{2}=1$ so that $\delta= \pm 1$.

Let $\eta$ be the 1 -form defined by $\eta(X)=\delta g(X, \xi)$ for any vector field $X$ on $M$, let $\phi$ be the $(1,1)$ tensor field defined by $\quad \phi\left(e_{1}\right)=e_{2}, \quad \phi\left(e_{2}\right)=-e_{1}, \quad \phi\left(e_{3}\right)=0$. Then by using the linearity of $\phi$ and $g$, we have $\phi^{2} X=-X+\eta(X) \xi$, with $\xi=e_{3}$. Further $g(\phi X, \phi Y)=g(X, Y)+\delta \eta(X) \eta(Y)$ for any vector fields $X$ and $Y$ on $M$. Hence for $e_{3}=\xi$, the structure defines an $(\delta)$-almost contact structure in $\mathbb{R}^{3}$.

Let $\nabla$ be the Levi-Civita connection with respect to the metric $g$, then we have

$$
\begin{aligned}
2 g\left(\nabla_{X} Y, Z\right)= & X g(Y, Z)+Y g(Z, X)-Z g(X, Y)-g(X,[Y, Z]) \\
& -g(Y,[X, Z])+g(Z,[X, Y]),
\end{aligned}
$$

which is know as Koszul's formula. Now we have

$$
\nabla_{e_{1}} e_{3}=-\frac{(\delta)}{z} e_{1}, \quad \nabla_{e_{2}} e_{3}=-\frac{(\delta)}{z} e_{2}, \quad \nabla_{e_{1}} e_{2}=0
$$

by using the above relation, for any vector $X$ on $M$, we have

$$
\nabla_{X} \xi=\delta[-\alpha \phi X-\beta(X+\eta(X) \xi)]
$$

where $\alpha=\frac{1}{z}$ and $\beta=-\frac{1}{z}$. Hence $(\phi, \xi, \eta, g, \delta)$ structure defines the $\delta$-Lorentzian trans-Sasakian structure in $\mathbb{R}^{3}$.

Here $\nabla$ be the Levi-Civita connection with respect to the metric $g$, then we have

$$
\left[e_{1}, e_{2}\right]=0, \quad\left[e_{1}, e_{3}\right]=-\frac{(\delta)}{z} e_{1}, \quad\left[e_{2}, e_{3}\right]=-\frac{(\delta)}{z} e_{2} .
$$

due to $g\left(e_{1}, e_{2}\right)=0$. Thus we have

$$
\begin{gathered}
\nabla_{e_{1}} e_{3}=-\frac{(\delta)}{z} e_{1}+e_{2}, \quad \nabla_{e_{1}} e_{2}=0 \\
\nabla_{e_{2}} e_{1}=0, \quad \nabla_{e_{2}} e_{2}=-\frac{(\delta)}{z} e_{2}, \quad \nabla_{e_{2}} e_{3}=-\frac{(\delta)}{z} e_{2}-e_{1} \quad \nabla_{e_{3}} e_{1}=0 \\
\nabla_{e_{3}} e_{2}=0, \quad \nabla_{e_{3}} e_{3}=-\frac{(\delta)}{z} e_{1}+e_{2} .
\end{gathered}
$$

The manifold $M$ satisfies (2.5) with $\alpha=\frac{1}{z}$ and $\beta=-\frac{1}{z}$. Hence $M$ is an $\delta$-Lorentzian 
trans-Sasakian manifolds. Then the non-vanishing components of the curvature tensor fields are computed as follows:

$$
\begin{gathered}
R\left(e_{1}, e_{3}\right) e_{3}=\frac{(\delta)}{z^{2}} e_{1}, \quad R\left(e_{3}, e_{1}\right) e_{3}=-\frac{(\delta)}{z^{2}} e_{1}, \\
R\left(e_{2}, e_{3}\right) e_{3}=\frac{(\delta)}{z^{2}} e_{1}, \quad R\left(e_{3}, e_{2}\right) e_{3}=-\frac{(\delta)}{z^{2}} e_{1} .
\end{gathered}
$$

From the above expression of the curvature tensor we can also obtain

$$
S\left(e_{1}, e_{1}\right)=S\left(e_{2}, e_{2}\right)=S\left(e_{3}, e_{3}\right)=\frac{\left(\delta^{2}\right)}{z^{2}}
$$

since $g\left(e_{1}, e_{3}\right)=g\left(e_{1}, e_{2}\right)=0$.

Therefore, we have

$$
S\left(e_{i}, e_{i}\right)=\frac{(\delta)}{z^{2}} g\left(e_{i}, e_{i}\right),
$$

for $i=1,2,3$, and $\alpha=\frac{1}{z}, \beta=-\frac{1}{z}$. Hence $M$ is also an Einstein manifold. In this case, from (3.11), we find $\lambda=\frac{(1+z \delta)}{z^{2}}$ and $\mu=\frac{(\delta)^{2}}{z}$, the data $(g, \xi, \lambda, \mu)$ is an expanding $\eta$-Ricci soliton on $(M, \phi, \xi, \eta, g)$.

\section{Gradient Ricci Solitons in 3-dimensional $\delta$-Lorentzian trans-Sasakian manifold}

If the vector field $V$ is the gradient of a potential function $\psi$ then $g$ is called a gradient Ricci soliton and (1.2) assume the form

$$
\nabla \nabla \psi=S+\lambda g
$$

This reduces to

$$
\nabla_{Y} D \psi=Q Y+\lambda Y
$$

where $D$ denoted the gradient operator of $g$. From (4.2) it follows

$$
R(X, Y) D \psi=\left(\bar{\nabla}_{X} Q\right) Y-\left(\bar{\nabla}_{Y} Q\right) X .
$$

Differentiating (3.20) we get

$$
\text { (4.4) } \begin{aligned}
\left(\nabla_{W} Q\right) X= & \left.\frac{d r(W)}{2}(X-\eta(X) \xi)\right)-\left(\frac{r}{2}-3\left(\alpha^{2}+\beta^{2}\right)\right)(\alpha(g(\phi W, X) \\
& +\beta \delta g(W, X)-\delta \beta \eta(X) \eta(W))+\eta(X) \nabla_{W} \xi .
\end{aligned}
$$

In (4.4) replacing $W=\xi$, we obtain

$$
\left.\left(\nabla_{\xi} Q\right) X=\frac{d r(\xi)}{2}(X-\eta(X) \xi)\right) .
$$


Then we have

$$
\begin{aligned}
g\left(\nabla_{\xi} Q\right) X-\left(\bar{\nabla}_{X} Q\right)(\xi, \xi) & =g\left(\frac{d r(\xi)}{2}(X-\eta(X) \xi, \xi)\right) \\
& \left.=\frac{d r(\xi)}{2}(g(X, \xi)-\eta(X))\right)=0 .
\end{aligned}
$$

Using (4.6) and (4.5), we obtain

$$
g(R(\xi, X) D \psi, \xi)=0 .
$$

From (2.12), we find

$$
g(\bar{R}(\xi, Y) D \psi, \xi)=\left(\alpha^{2}+\beta^{2}\right)(g(Y, D \psi)-\eta(Y) \eta(D \psi)) .
$$

Using (11.7), we get

$$
\begin{gathered}
\left(\alpha^{2}+\beta^{2}\right)(g(Y, D \psi)-\eta(Y) \eta(D \psi))=0 \\
\left(\alpha^{2}+\beta^{2}\right)(g(Y, D \psi)-\eta(Y) g(D \psi, \xi))=0,
\end{gathered}
$$

or

$$
(g(Y, D \psi)-g(Y, \xi) g(D \psi, \xi))=0,
$$

which implies

$$
D \psi=(\xi \psi) \xi, \quad \text { since } \quad \alpha^{2}+\beta^{2} \neq-\delta(\xi \beta) .
$$

Now, using (4.8) and (4.2), we get

$$
\begin{array}{r}
S(X, Y)+\lambda g(X, Y)=g\left(\nabla_{Y} D \psi, X\right)=g\left(\nabla_{Y}(\xi \psi) \xi, X\right) \\
=(\xi \psi) g\left(\bar{\nabla}_{Y} \xi, X\right)+Y(\xi \psi) \eta(X) \\
=(\xi \psi) g(-\delta \alpha \phi Y-\delta \beta Y-\delta \beta \eta(Y) \xi, X)+Y(\xi \psi) \eta(X)
\end{array}
$$

$$
\begin{array}{r}
S(X, Y)+\lambda g(X, Y)=-\delta \alpha(\xi \psi) g(\phi Y, X)-\delta \beta(\xi \psi) g(Y, X) \\
-\delta \beta(\xi \psi) \eta(Y) \eta(X)+Y(\xi \psi) \eta(X) .
\end{array}
$$

Putting $X=\xi$ in (4.9) and using (2.15) we get

(4.10) $\bar{S}(Y, \xi)+\lambda \eta(Y)=Y(\xi \psi)=\left[\lambda+2 \delta \beta+2\left(\alpha^{2}+\beta^{2}-\delta(\xi \beta)\right)\right] \eta(Y)$. 
Interchanging $X$ and $Y$ in (4.9), we get

$$
\begin{aligned}
S(X, Y)+\lambda g(X, Y)= & -\delta \alpha(\xi \psi) g(Y, \phi X)-\delta \beta(\xi \psi) g(X, Y) \\
& -\delta \beta(\xi \psi) \eta(Y) \eta(X)+X(\xi \psi) \eta(Y) .
\end{aligned}
$$

Adding (4.9) and (4.11) we get

$$
\begin{aligned}
2 S(X, Y)+2 \lambda g(X, Y) & =-2 \delta \beta(\xi \psi) g(X, Y)+Y(\xi \psi) \eta(X) \\
& -2 \delta \beta(\xi \psi) \eta(X) \eta(Y)+X(\xi \psi) \eta(Y) .
\end{aligned}
$$

Using (4.10) in (4.12) we have

$$
\begin{aligned}
S(X, Y)+\lambda g(X, Y)= & -\delta \beta(\xi \psi)[g(X, Y)-\eta(X) \eta(Y)] \\
& +\left[\lambda+\delta \beta+2\left(\alpha^{2}+\beta^{2}-\delta(\xi \beta)\right)\right] \eta(X) \eta(Y)
\end{aligned}
$$

Then using (4.2) we have

$$
\begin{aligned}
\nabla_{Y} D \psi= & -\delta \beta(\xi \psi)(Y-\eta(Y) \xi) \\
& +\left[\lambda+\delta \beta+2\left(\alpha^{2}+\beta^{2}-\delta(\xi \beta)\right)\right] \eta(Y) \xi
\end{aligned}
$$

Using (11.14) we calculate

$$
\begin{gathered}
R(X, Y) D \psi=\nabla_{X} \nabla_{Y} D \psi-\nabla_{Y} \nabla_{X} D \psi-\nabla_{[X, Y]} D \psi \\
=-\delta \beta X(\xi \psi) Y+\delta \beta Y(\xi \psi) X \\
-\delta \beta Y(\xi \psi) \eta(X) \xi+\delta \beta X(\xi \psi) \eta(Y) \xi \\
+\left[\lambda+\delta \beta+2\left(\alpha^{2}+\beta^{2}-\delta(\xi \beta)\right)\right]\left(\left(\nabla_{X} \eta\right)(Y) \xi-\left(\nabla_{Y} \eta\right)(X) \xi\right) \\
+\left[\lambda+\delta \beta+2\left(\alpha^{2}+\beta^{2}-\delta(\xi \beta)\right)\right]\left(\left(\nabla_{X} \xi\right) \eta(Y) \xi-\left(\nabla_{Y} \xi\right) \eta(X)\right) .
\end{gathered}
$$

Taking inner product with $\xi$ in $(4.15)$, we get

$$
0=g((X, Y) D \psi, \xi)=2 \delta \alpha\left[\lambda+\delta \beta+2\left(\alpha^{2}+\beta^{2}-\delta(\xi \beta)\right)\right] g(\phi Y, X) .
$$

Thus we have $2 \delta \alpha\left[\lambda+\delta \beta+2\left(\alpha^{2}+\beta^{2}-\delta(\xi \beta)\right)\right]=0$.

Now we consider the following cases:

Case (i) $\delta \alpha=0$, or

Case (ii) $\left[\lambda+\delta \beta+2\left(\alpha^{2}+\beta^{2}-\delta(\xi \beta)\right)\right]=0$,

Case (iii) $\alpha=0$ and $\left[\lambda+\delta \beta+2\left(\alpha^{2}+\beta^{2}-\delta(\xi \beta)\right)\right]=0$. 
In this case, we have the following;

Case (i) If $\alpha=0$, the manifold reduces to a $\delta$-Lorentzian $\beta$-Kenmotsu manifold.

Case (ii) Let $\left[\lambda+\delta \beta+2\left(\alpha^{2}+\beta^{2}-\delta(\xi \beta)\right)\right]=0$. If we use this in (4.10) we get $Y(\xi \psi)=-\delta \beta(\xi \psi) \eta(Y)$. Substitute this value in (11.12) we obtain

$$
S(X, Y)+\lambda g(X, Y)=-\delta \beta(\xi \psi) g(X, Y)-2 \delta \beta \eta(X) \eta(Y) .
$$

Now, contracting (4.17), we get

$$
r+3 \lambda=-3 \delta \beta(\xi \psi)-2 \delta \beta,
$$

which implies

$$
(\xi \psi)=\frac{r}{-3 \delta \beta}+\frac{\lambda}{-\delta \beta}+\frac{2}{-3} .
$$

If $r=$ constant, then $(\xi \psi)=$ constant $=k($ say $)$. Therefore from (4.8) we have $D \psi=(\xi \psi) \xi=k \xi$. This we can write this equation as

$$
g(D \psi, X)=k \eta(X)
$$

which means that $d \psi(X)=k \eta(X)$. Applying $d$ this, we get $k d \eta=0$. Since $d \eta \neq 0$, we have $k=0$. Hence we get $D \psi=0$. This means that $\psi=$ constant Therefore equation (11.1) reduces to

$$
S(X, Y)=2\left(\alpha^{2}+\beta^{2}-\delta(\xi \beta)\right) g(X, Y),
$$

that is $M$ is an Einstein manifold.

Case (iii) Using $\alpha=0$ and $\left[\lambda+\delta \beta+2\left(\alpha^{2}+\beta^{2}-\delta(\xi \beta)\right)\right]=0$. in (4.10) we obtain $Y(\xi \psi)=-\delta \beta(\xi \psi) \eta(Y)$. Now as in Case (ii) we conclude that the manifold is an Einstein manifold.

Thus we have the following :

Theorem 4.1. If a 3-dimensional $\delta$-Lorentzian trans-Sasakian manifold with constant scalar curvature admits gradient Ricci soliton, then the manifold is either a $\delta$ Lorentzian $\beta$-Kenmotsu manifold or an Einstein manifold provided $\alpha, \beta=$ constant.

In [9], it was proved that if a 3-dimensional compact connected trans-Sasakian manifold is of constant curvature, then it is either $\alpha$-Sasakian or $\beta$-Kenmotsu. Since for a 3-dimensional Riemannian manifold constant curvature and Einstein manifold are equivalent, therefore from the Theorem 3 we state the following:

Corollary 4.1. If a compact 3-dimensional $\delta$-Lorentzian trans-Sasakian manifold with constant scalar curvature admits Ricci soliton, then the manifold is either $\delta$ Lorentzian $\alpha$-Sasakian or $\delta$-Lorentzian $\beta$-Kenmotsu. 
Also in [13], authors proved that a 3-dimensional connected trans-Sasakian manifold is locally $\phi$-symmetric if and only if the scalar curvature is constant provided $\alpha$ and $\beta$ are constants. Hence, from Theorem 3 we obtain the following:

Corollary 4.2. If a locally $\phi$-symmetric 3-dimensional connected $\delta$-Lorentzian trans-Sasakian manifold its admits gradient Ricci soliton, then manifold is either $\delta$-Lorentzian $\beta$-Kenmotsu or Einstein manifold provided $\alpha, \beta=$ constant.

\section{Acknowledgement}

The author is grateful to the referee for his/her valuable comments and suggestions.

\section{REFEREN CES}

1. S. M. Bhati: On weakly Ricci $\phi$-symmetric $\delta$-Lorentzian trans-Sasakian manifolds. Bull. Math. Anal. Appl., 5 (1), (2013), 36-43.

2. D. E. Blair and J. A. OuBinA: Conformal and related changes of metric on the product of two almost contact metric manifolds. Publ. Mat. 34 (1990), 199-207.

3. A. Bejancu and K. L. Duggal: Real hypersurfaces of indefnite Kaehler manifolds. Int. J. Math and Math Sci., 16 (3) (1993), 545-556.

4. A. M. Blaga: $\eta$-Ricci solitons on Lorentzian para-Sasakian manifolds. Filomat 30 (2) (2016), 489-496.

5. A. M. BlaGA: $\eta-$ Ricci solitons on para-Kenmotsu manifolds. Balkan J. Geom. Appl. 20 (2015), 1-13.

6. C. S. Bagewadi and G. Ingalahalli: Ricci solitons in $(\epsilon, \delta)$-trans-Sasakain manifolds. Int. J. Anal.Apply., 2 (2017), 209-217.

7. C. Calin and M. Crasmareanu: $\eta$-Ricci solitons on Hopf hypersurfaces in complex space forms. Rev. Roumaine Math. Pures Appl. 57(1) (2012), 55-63.

8. J. T. Сho and M. Kimura: Ricci solitons and Real hypersurfaces in a complex space form. Tohoku math.J., 61(2009), 205-212.

9. U. C. De and A. SARKAR: On $(\epsilon)$-Kenmotsu manifolds. Hadronic J. 32 (2009), 231-242.

10. U. C. DE and A. SARKAR: On three-dimensional trans-Sasakian Manifolds. Extracta Math. 23 (2008) 265-277.

11. U. C. DE and K. DE: On Lorentzian trans-Sasakian manifolds. Commun. Fac. Sci. Univ. Ank. series A1, 62 (2), (2013), 37-51.

12. L. P. Eisenhart: Symmetric tensors of the second order whose first covariant derivatives are zero. Trans. Amer. Math. Soc., 25 (2) (1923), 297-306.

13. A. Gray and L. M. Harvella: The sixteen classes of almost Hermitian manifolds and their linear invariants. Ann. Mat. Pura Appl., 123 (4) (1980), 35-58.

14. R. S. Hamilton: The Ricci flow on surfaces, Mathematics and general relativity (Santa Cruz. CA, 1986), Contemp. Math. 71, Amer. Math. Soc., (1988), 237-262.

15. G. Ingalahalli and C. S. BAGewADI: Ricci solitons in $(\epsilon)$-trans-Sasakian manifolds. J. Tensor Soc. 6 (1) (2012), 145-159. 
16. T. IKAWA and M. ERdogAn: Sasakian manifolds with Lorentzian metric. Kyungpook Math.J. 35(1996), 517-526.

17. H. LEVY: Symmetric tensors of the second order whose covariant derivatives vanish. Ann. Math. 27(2) (1925), 91-98.

18. J. C. Marrero: The local structure of trans-Sasakian manifolds. Annali di Mat. Pura ed Appl. 162 (1992), 77-86.

19. K. Matsumoto: On Lorentzian paracontact manifolds. Bull. Yamagata Univ. Nat. Science, 2 (1989), 151-156.

20. I. Minai, A. OiAgA and R. RoscA: Lorentzian Kenmotsu manifolds having two skewsymmetric conformal vector fields. Bull. Math. Soc. Sci. Math. Roumania, 42 (1999), $237-251$.

21. J. A. Oubina: New classes of almost contact metric structures. Publ. Math. Debrecen 32 (1985), 187-193.

22. S. S. PUJAR and V. J. KhaIRNAR: On Lorentzian trans-Sasakian manifold-I. Int.J.of Ultra Sciences of Physical Sciences, 23(1) (2011), 53-66.

23. S. S. PUJAR: On $\delta$-Lorentzian $\alpha$-Sasakian manifolds, to appear in Antarctica J. of Mathematics 8(2012).

24. R. Sharma: Certain results on $K$-contact and $(k, \mu)$-contact manifolds. J. Geom., 89(1-2) (2008), 138-147.

25. S. S. Shukla and D. D. Singh: On $(\epsilon)$-trans-Sasakian manifolds. Int. J. Math. Anal. 49(4) (2010), 2401-2414.

26. M. D. SiddiqI, A. Haseeb and M. Ahmad: A Note On Generalized Ricci-Recurrent $(\epsilon, \delta)$-trans-Sasakian Manifolds. Palestine J. Math., 4(1) (2015), 156-163.

27. M. D. SIDDIQI: $\eta$-Ricci soliton3 in 3-dimensional normal almost contact metric manifolds. Bull. Transilvania Univ. Brasov, Series III: Math, Informatics, Physics, 11(60) (2018), 215-234.

28. M. D. SiddiqI: $\eta$-Ricci soliton in $(\varepsilon, \delta)$-trans-Sasakian manifolds. Facta. Univ. (Nis), Math. Inform., 34(1) (2019), 45-56.

29. M. D. SidDIQI: Generalized $\eta-$ Ricci solitons on trans-Sasakian manifolds. Eurasian Bull. Math. EBM 1(3) (2018), 107-116.

30. M. D. SidDiQI: Generalized Ricci Solitons on trans-Sasakian Manifolds. Khayyam Journal of Math. 4(2) (2018), 178-186.

31. M. Turan, U. C. DE and A. Yildiz: Ricci solitons and gradient Ricci solitons on 3-dimensional trans-Sasakian manifolds. Filomat, 26(2) (2012), 363-370.

32. T. TAKahashi: Sasakian manifolds with Pseudo-Riemannian metric. Tohoku Math. J. 21 (1969), 271-290.

33. M. M. Tripathi, E. Kiliç and S. Y. PerkTAŞ: Indefinite almost metric manifolds. Int.J. of Math. and Mathematical Sciences, (2010) Article ID 846195, doi.10,1155/846195.

34. S. TANnO: The automorphism groups of almost contact Riemannian manifolds. Tohoku Math.J. 21 (1969), 21-38.

35. K. Vinu and H. G. NAGARAJA: $\eta$-Ricci solitons in trans-Sasakian manifolds. Commun. Fac. sci. Univ. Ank. Series A1, 66(2) (2017), 218-224.

36. X. Xufeng and C. XiaOli: Two theorems on $(\epsilon)$-Sasakain manifolds. Int. J. Math. Math.Sci., 21(2) (1998), 249-254. 
37. A. F. YAliniz, A. YILdiz and M. Turan: On three-dimensional Lorentzian $\beta$-Kenmotsu manifolds. Kuwait J. Sci. Eng. 36 (2009), 51-62.

38. A. Yildiz, M. Turan and C. Murathan: A class of Lorentzian $\alpha-$ Sasakian manifolds. Kyungpook Math. J. 49 (2009), 789 -799. 